\title{
Analysis and Design of Robust Positivity and Stability for Continuous-Time Linear Uncertain Systems
}

\author{
Kuo-Liang Yen, ${ }^{1}$ Kuo-Shong Wang, ${ }^{1}$ and Yau-Tarng Juang ${ }^{2}$ \\ ${ }^{1}$ Mechanical Engineering, National Central University, Taoyuan, Zhongli 320, Taiwan \\ ${ }^{2}$ Electrical Engineering, National Central University, Taoyuan, Zhongli 320, Taiwan \\ Correspondence should be addressed to Kuo-Liang Yen; bouliang@pchome.com.tw \\ Received 17 January 2015; Revised 19 May 2015; Accepted 24 June 2015 \\ Academic Editor: Dan Huang
}

Copyright (c) 2015 Kuo-Liang Yen et al. This is an open access article distributed under the Creative Commons Attribution License, which permits unrestricted use, distribution, and reproduction in any medium, provided the original work is properly cited.

\begin{abstract}
This paper deals with the analysis and design of positivity and stability of linear continuous conic systems. First, two robustness analysis theorems are proposed for the systems with state-feedback. Second, the state-feedback stabilization problem is solved by using linear programming (LP). Numerical examples are given for illustration. Finally, the conclusions are made.
\end{abstract}

\section{Introduction}

Many researchers study positive systems. The design of observers and dynamic output-feedback controllers for positive linear systems with interval uncertainties was finished by Shu et al. [1]. The continuous-time case and the discrete-time case were both treated in a unified linear matrix inequality (LMI) framework. Liu et al. [2] tried to adapt the linear copositive Lyapunov function to LTI discrete-time positive systems with delays, focusing on controller design under the positivity constraint and/or with bounds on the inputs and the states. Liu [3] finished the continuous-time positive systems with delays. Roszak and Davison [4] considered the problem of stabilization via output-feedback, state-feedback, and stabilization via the sequential separation principle for positive linear time-invariant (LTI) systems. Jacquez and Simon [5] applied compartmental systems in different fields ranging from biology and chemistry, over ecology to economy and sociology. Marchetti et al. [6] proposed an improved PID control strategy for blood glucose control. This is a great contribution for type 1 diabetes. Rami and Tadeo [7] pointed out that linear programming (LP) approach is simpler than the linear matrix inequality (LMI) approach and possesses a low computational complexity. They also solved an important synthesis problem: finding bounded stabilizing state-feedback control. In this paper, we will solve the statefeedback stabilization problems for positive linear systems with conic uncertainty in terms of linear programming (LP).

\section{Preliminary}

Consider the following linear system:

$$
\dot{x}=A x,
$$

where $x(t) \in R^{n}$ is the system state and $A \in R^{n \times n}$. The system in (1) is said to be positive if and only if, for any nonnegative initial condition, denoted as $x(0)=x_{0} \geq 0$, the resulting state should be nonnegative; that is, $x(t) \geq 0$ for all $t>0$.

Definition 1. $A \in R^{n \times n}$ is said to be Metzler if all off-diagonal entries are nonnegative.

\section{Positivity and Stability of Continuous-Time Conic Linear Systems}

Lemma 2 (see [8]). The system (1) is a continuous-time positive system if and only if the matrix $A$ is a Metzler matrix. 
Lemma 3 (see [7]). Continuous-time positive linear system is asymptotically stable if and only if there exists a $d \in R_{+}^{n}$ with Ad $<0$.

Consider an autonomous system with the system matrix $A$ represented by a more general conic form:

$$
\begin{aligned}
\dot{x}(t) & =A x(t), \\
x(0) & =x_{0} \in R_{+}^{n}, \\
A & \in\left\{\sum_{p=1}^{\bar{p}} \alpha_{p} A_{p} \mid \alpha_{p} \geq 0, \sum_{p=1}^{\bar{p}} \alpha_{p} \neq 0\right\},
\end{aligned}
$$

where $A_{p}$ are given constant matrices and $\bar{p}$ is a given number. Then, the following result is obtained.

Theorem 4. The linear conic system in (2) is positive and asymptotically stable if $A_{p}$ are Metzler matrices and there exists ad $\in R_{+}^{n}$ such that $A_{p} d<0$, for all $p=1, \ldots, \bar{p}$.

Proof. Owing to the matrices $A_{p}$ are Metzler; let $a_{p, i j}$ and $a_{i j}$ be the entries of $A_{p}$ and, for $1 \leq i \neq j \leq n, p=1, \ldots, \bar{p}$. From (2), $a_{i j}=\sum_{p=1}^{\bar{p}} \alpha_{p} a_{p, i j} \geq 0$, for $1 \leq i \neq j \leq n$. This shows that the off-diagonal entries of $A$ are nonnegative. By Lemma 2, the system (2) is positive. On the other hand, $A_{p} d<0$, for $p=$ $1, \ldots, \bar{p}$; that is, $\sum_{j=1}^{n} a_{p, i j} d_{j}<0$, for $1 \leq i \leq n, p=1, \ldots, \bar{p}$. From (2), $A d=\sum_{j=1}^{n} \sum_{p=1}^{\bar{p}} \alpha_{p} a_{p, i j} d_{j}<0$, for $1 \leq i \leq n$. By Lemma 3 , system (2) is asymptotically stable.

Remark 5. The polytopic and interval representations are the special cases of the considered conic set representation.

In the following, consider the continuous-time linear conic system:

$$
\begin{aligned}
\dot{x}(t) & =A x(t)+B u(t), \\
x(0) & =x_{0} \in R_{+}^{n}, \\
A & \in\left\{\sum_{p=1}^{\bar{p}} \alpha_{p} A_{p} \mid \alpha_{p} \geq 0, \sum_{p=1}^{\bar{p}} \alpha_{p} \neq 0\right\}, \\
B & \in\left\{\sum_{q=1}^{\bar{q}} \beta_{q} B_{q} \mid \beta_{q} \geq 0, \sum_{q=1}^{\bar{q}} \beta_{q} \neq 0\right\},
\end{aligned}
$$

where $A_{p}=\left[a_{p, i j}\right]_{n \times n}$ and $B_{q}=\left[b_{q, i h}\right]_{n \times m}$ are given constant matrices and $\bar{p}$ and $\bar{q}$ are given constants. When the statefeedback $u=K x$, where $K=\left[k_{h j}\right] \in R^{m \times n}$, is applied, the closed-loop system becomes

$$
\begin{aligned}
& A \in\left\{\sum_{p=1}^{\bar{p}} \alpha_{p} A_{p} \mid \alpha_{p} \geq 0, \sum_{p=1}^{\bar{p}} \alpha_{p} \neq 0\right\}, \\
& B \in\left\{\sum_{q=1}^{\bar{q}} \beta_{q} B_{q} \mid \beta_{q} \geq 0, \sum_{q=1}^{\bar{q}} \beta_{q} \neq 0\right\} .
\end{aligned}
$$

Lemma 6. System (4) is positive and asymptotically stable if $A_{p}+B_{q} K$ are Metzler matrices and there exists a $d \in R_{+}^{n}$ such that $\left(A_{p}+B_{q} K\right) d<0$, for $p=1, \ldots, \bar{p}, q=1, \ldots, \bar{q}$.

Proof. By Theorem 4, the system in (2) is positive and asymptotically stable if $A_{p}$ are Metzler matrices and there exists a $d \in R_{+}^{n}$ such that $A_{p} d<0$, for all $p=1, \ldots, \bar{p}$.

Now, in system (4), assume that $A_{\mathrm{cl}}=A+B K$ where

$$
\begin{aligned}
& A \in\left\{\sum_{p=1}^{\bar{p}} \alpha_{p} A_{p} \mid \alpha_{p} \geq 0, \sum_{p=1}^{\bar{p}} \alpha_{p} \neq 0\right\}, \\
& B \in\left\{\sum_{q=1}^{\bar{q}} \beta_{q} B_{q} \mid \beta_{q} \geq 0, \sum_{q=1}^{\bar{q}} \beta_{q} \neq 0\right\} .
\end{aligned}
$$

Then $A_{\mathrm{cl}}=A+B K=\sum_{q=1}^{\bar{q}} \sum_{p=1}^{\bar{p}} \alpha_{p} \beta_{q}\left(A_{p}+B_{q} K\right)$; that is, $A_{\mathrm{cl}}$ has $\bar{p} \bar{q}$ vertices. Hence, system (4) is positive and asymptotically stable if $A_{p}+B_{q} K$ are Metzler matrices and there exists a $d \in R_{+}^{n}$ such that $\left(A_{p}+B_{q} K\right) d<0$, for $p=1, \ldots, \bar{p}, q=1, \ldots, \bar{q}$.

Theorem 7. Using the state-feedback $u=K x$ with $K=$ $\left[k_{h j}\right] \in R^{m \times n}$, the closed-loop system in (4) is positive and asymptotically stable if there exist $a\left[d_{j}\right] \in R_{+}^{n}$ and $a\left[z_{h j}\right] \in$ $R^{m \times n}$ such that, for all $p=1, \ldots, \bar{p}$ and $q=1, \ldots, \bar{q}$,

$$
\begin{aligned}
a_{p, i j} d_{j}+\sum_{h=1}^{m} b_{q, i h} z_{h j} \geq 0, & 1 \leq i \neq j \leq n, \\
\sum_{j=1}^{n}\left(a_{p, i j} d_{j}+\sum_{h=1}^{m} b_{q, i h} z_{h j}\right)<0, & 1 \leq i \leq n,
\end{aligned}
$$

where $K=Z D^{-1}, K=\left[k_{h j}\right] \in R^{m \times n}$, and

$$
D=\operatorname{diag}\left\{d_{1}, d_{2}, \ldots, d_{n}\right\}
$$

Proof. (a) Positivity is as follows.

Due to

$$
a_{p, i j} d_{j}+\sum_{h=1}^{m} b_{q, i h} z_{h j} \geq 0, \quad d_{j}>0
$$

owing to $k_{h j}=z_{h j} d_{j}^{-1}$,

$$
a_{p, i j}+\sum_{h=1}^{m} b_{q, i h} k_{h j} \geq 0
$$


for $1 \leq i \neq j \leq n, p=1, \ldots, \bar{p}$, and $q=1, \ldots, \bar{q}$, this shows that the off-diagonal entries of the matrices $A_{p}+B_{q} K$ are nonnegative. By Lemma 6, the closed-loop system in (4) is positive.

(b) Stability is as follows.

Due to

$$
\sum_{j=1}^{n}\left(a_{p, i j} d_{j}+\sum_{h=1}^{m} b_{q, i h} z_{h j}\right)<0, \quad d_{j}>0,
$$

owing to $k_{h j}=z_{h j} d_{j}^{-1}$,

$$
\sum_{j=1}^{n}\left(a_{p, i j}+\sum_{h=1}^{m} b_{q, i h} k_{h j}\right) d_{j}<0
$$

for $1 \leq i \leq n, p=1, \ldots, \bar{p}$, and $q=1, \ldots, \bar{q}$, this shows that there exists a $\left[d_{j}\right] \in R_{+}^{n}$ such that $\left(A_{p}+B_{q} K\right) d<0$. By Lemma 6, the closed-loop system in (4) is asymptotically stable.

Remark 8. Since the conditions in $((6 a),(6 b))$ are linear in the entries of $\left[d_{j}\right]$ and $\left[z_{h j}\right]$, these variables can be easily solved by using the linear programming. After $\left[d_{j}\right]$ and $\left[z_{h j}\right]$ are decided, the robust state-feedback is determined by $K=$ $Z D^{-1}$. This will accomplish the design for the positivity and stability of continuous-time conic positive linear systems.

\section{Numerical Examples}

In this section, the corresponding example is presented to show the effectiveness of our results.

Example 1. Consider a continuous-time conic system described by (3). Let $\bar{p}=4$ and $\bar{q}=4$; the matrices $A=$ $\sum_{p=1}^{4} \alpha_{p} A_{p}$ and $B=\sum_{q=1}^{4} \beta_{q} B_{q}$ with the following parame-
ters:

$$
\begin{aligned}
& A_{1}=\left[\begin{array}{cc}
-0.5 & -0.4 \\
0.6 & -0.3
\end{array}\right], \\
& A_{2}=\left[\begin{array}{cc}
-0.7 & 0.6 \\
0.9 & 0.5
\end{array}\right], \\
& A_{3}=\left[\begin{array}{cc}
-0.3 & 0.5 \\
-0.7 & -0.5
\end{array}\right], \\
& A_{4}=\left[\begin{array}{cc}
-0.9 & 0.4 \\
0.6 & 0.3
\end{array}\right], \\
& B_{1}=\left[\begin{array}{cc}
0.5 & 0.2 \\
0.7 & -0.4
\end{array}\right],
\end{aligned}
$$

$$
\begin{aligned}
B_{2} & =\left[\begin{array}{cc}
0.4 & 0.5 \\
0.3 & -0.5
\end{array}\right], \\
B_{3} & =\left[\begin{array}{cc}
0.3 & 0.4 \\
0.6 & -0.3
\end{array}\right], \\
B_{4} & =\left[\begin{array}{cc}
0.6 & 0.3 \\
0.4 & -0.2
\end{array}\right], \\
\alpha_{1} & \geq 0, \\
\alpha_{2} & \geq 0, \\
\alpha_{3} & \geq 0, \\
\alpha_{4} & \geq 0, \\
\sum_{p=1}^{4} \alpha_{p} & =4, \\
\beta_{1} & \geq 0, \\
\beta_{2} & \geq 0, \\
\beta_{3} & \geq 0, \\
\beta_{4} & \geq 0, \\
\sum_{q=1}^{4} \beta_{q} & =4 .
\end{aligned}
$$

By Theorem 7 and MATLAB to get

$$
\begin{aligned}
Z & =\left[\begin{array}{cc}
-8.7788 & -16.7758 \\
-57.8970 & 72.5071
\end{array}\right], \\
d & =\left[\begin{array}{l}
8.2047 \\
4.3698
\end{array}\right], \\
K & =Z D^{-1}=\left[\begin{array}{ll}
-1.0700 & -3.8390 \\
-7.0566 & 16.5926
\end{array}\right] .
\end{aligned}
$$

For the uncertain parameters $\alpha_{1}, \alpha_{2}, \alpha_{3}, \alpha_{4}, \beta_{1}, \beta_{2}, \beta_{3}$, and $\beta_{4}$, the following cases are presented.

Case $1\left(\alpha_{1}=0, \alpha_{2}=2, \alpha_{3}=0, \alpha_{4}=2, \beta_{1}=2, \beta_{2}=0, \beta_{3}=\right.$ 2 , and $\beta_{4}=0$ ). The matrix of the closed-loop system (4) is obtained as

$$
A+B K=\left[\begin{array}{cc}
-13.3798 & 15.7687 \\
10.0972 & -31.6110
\end{array}\right] \text {. }
$$

Case $2\left(\alpha_{1}=1, \alpha_{2}=1, \alpha_{3}=1, \alpha_{4}=1, \beta_{1}=1, \beta_{2}=1, \beta_{3}=\right.$ 1 , and $\beta_{4}=1$ ). The matrix of the closed-loop system (4) is obtained as

$$
A+B K=\left[\begin{array}{cc}
-14.2051 & 17.4195 \\
9.1392 & -30.9076
\end{array}\right] \text {. }
$$




\section{Conclusion}

The proofs of the two theorems are finished. The simulations are complete by using Theorem 7. All results are Hurwitz matrix. In short, the systems are positive and asymptotically stable.

\section{Conflict of Interests}

The authors declare that there is no conflict of interests regarding the publication of this paper.

\section{References}

[1] Z. Shu, J. Lam, H. Gao, B. Du, and L. Wu, "Positive observers and dynamic output-feedback controllers for interval positive linear systems," IEEE Transactions on Circuits and Systems. I. Regular Papers, vol. 55, no. 10, pp. 3209-3222, 2008.

[2] X. Liu, L. Wang, W. Yu, and S. Zhong, "Constrained control of positive discrete-time systems with delays," IEEE Transactions on Circuits and Systems II: Express Briefs, vol. 55, no. 2, pp. 193197, 2008.

[3] X. Liu, "Constrained control of positive systems with delays," IEEE Transactions on Automatic Control, vol. 54, no. 7, pp. 1596$1600,2009$.

[4] B. Roszak and E. J. Davison, "Necessary and sufficient conditions for stabilizability of positive LTI systems," Systems \& Control Letters, vol. 58, no. 7, pp. 474-481, 2009.

[5] J. A. Jacquez and C. P. Simon, "Qualitative theory of compartmental systems," The SIAM Review, vol. 35, no. 1, pp. 43-79, 1993.

[6] G. Marchetti, M. Barolo, L. Jovanovic, H. Zisser, and D. E. Seborg, "An improved PID switching control strategy for type 1 diabetes," IEEE Transactions on Biomedical Engineering, vol. 55 , no. 3, pp. 857-865, 2008.

[7] M. A. Rami and F. Tadeo, "Controller synthesis for positive linear systems with bounded controls," IEEE Transactions on Circuits and Systems II: Express Briefs, vol. 54, no. 2, pp. 151-155, 2007.

[8] B. Roszak and E. J. Davison, "The multivariable servomechanism problem for positive LTI systems," IEEE Transactions on Automatic Control, vol. 55, no. 9, pp. 2204-2208, 2010. 


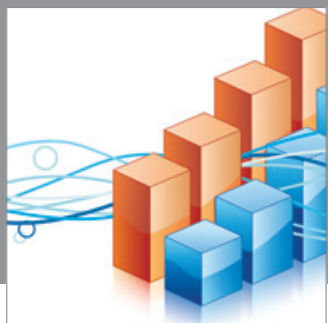

Advances in

Operations Research

mansans

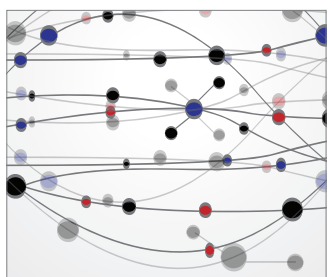

The Scientific World Journal
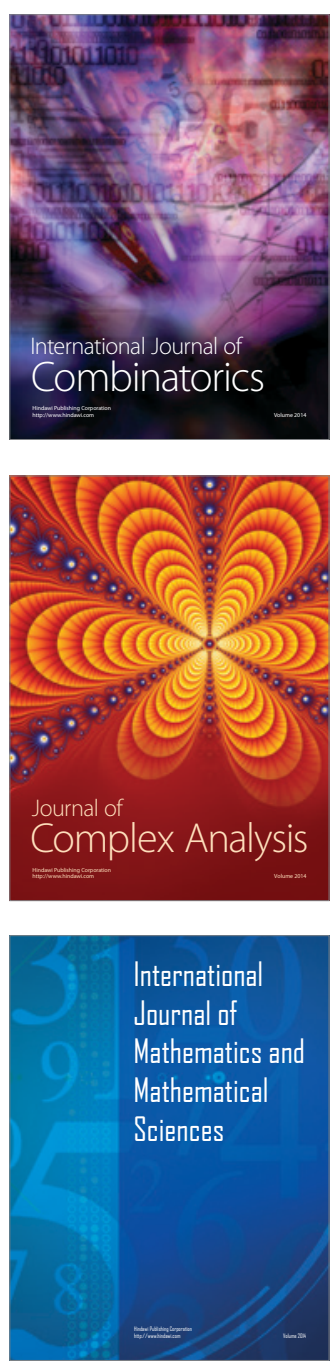
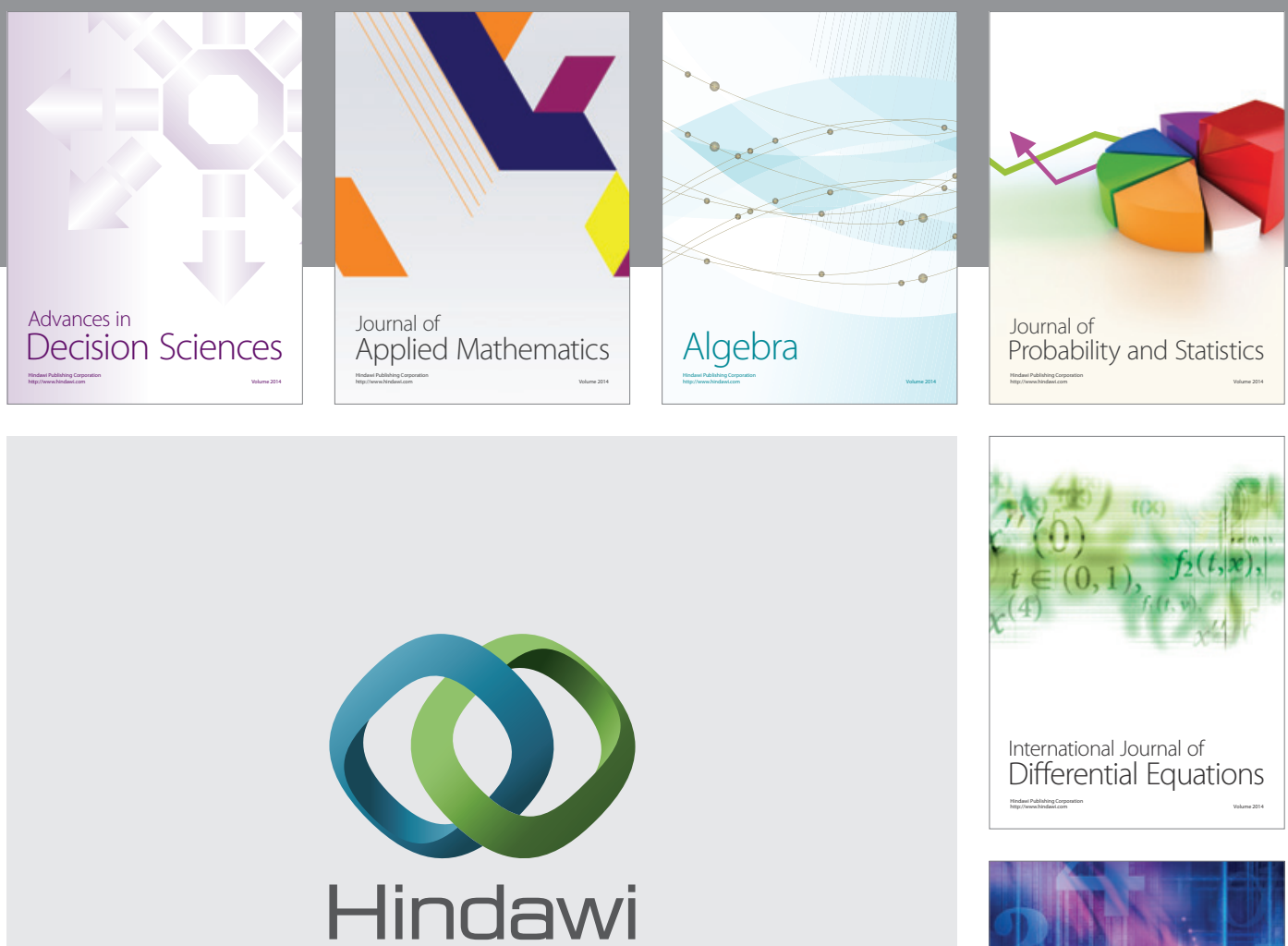

Submit your manuscripts at http://www.hindawi.com
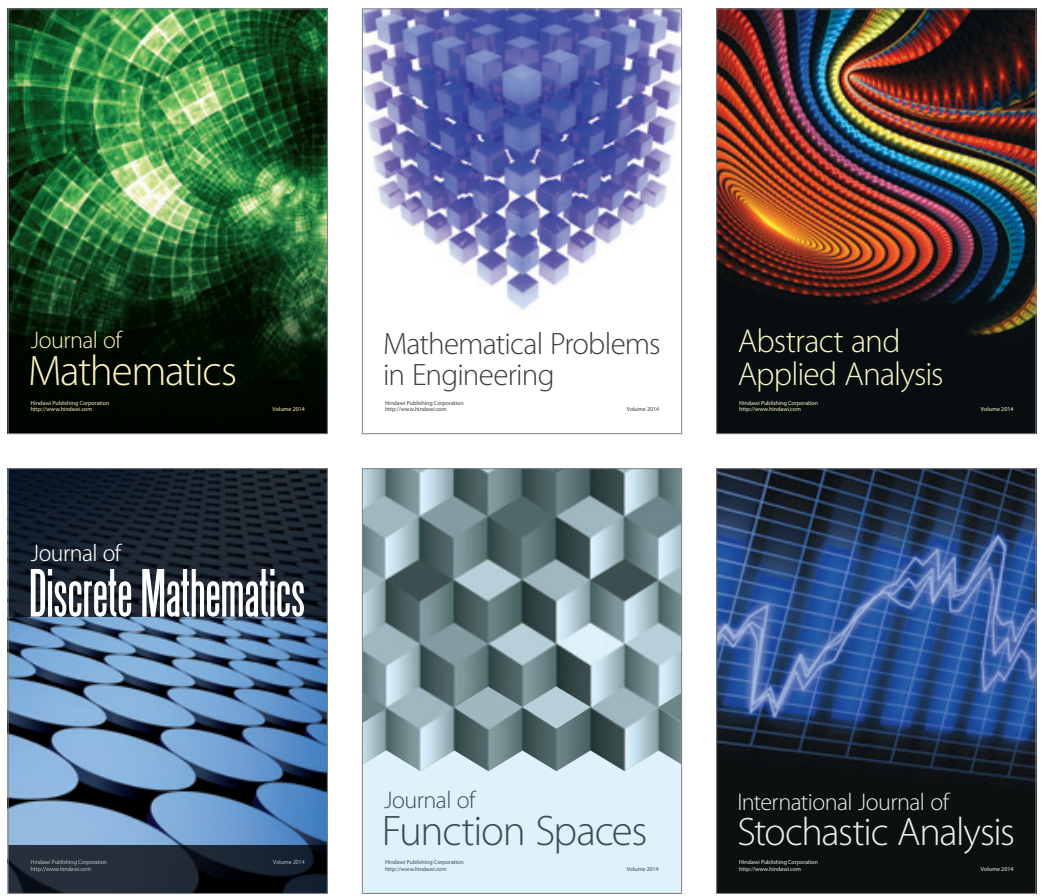

Journal of

Function Spaces

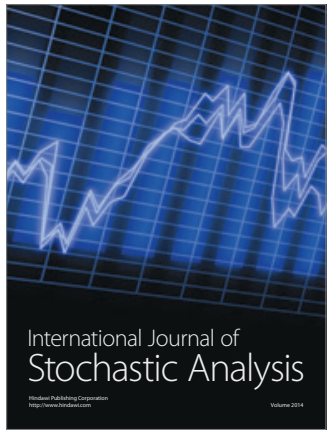

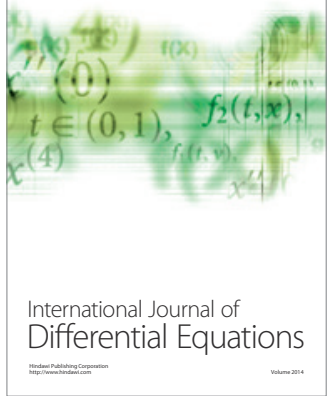
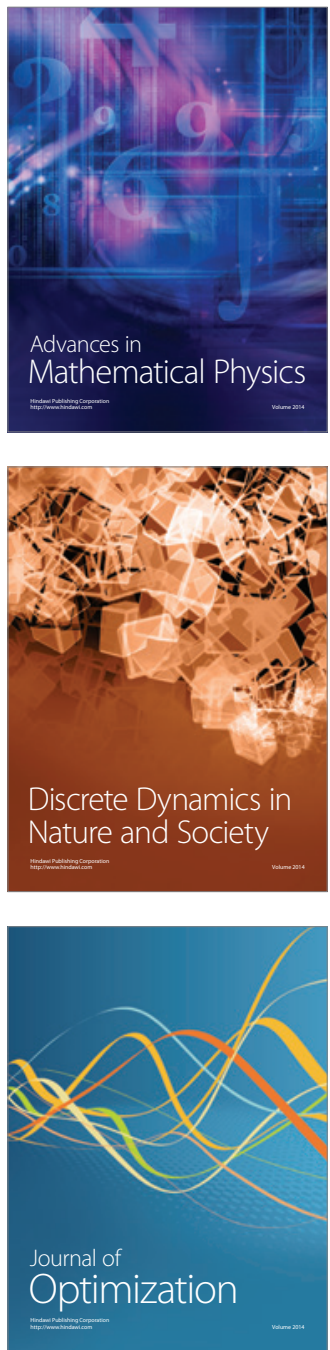